\title{
Les salariés agricoles. Entre ancrage sectoriel et précarité
}

Agricultural employees: between sectoral attachment and precarity

\section{Sonia Bellit and Cécile Détang-Dessendre}

\section{(2) OpenEdition}

1 Journals

\section{Electronic version}

URL: http://journals.openedition.org/economierurale/4373

DOI: 10.4000/economierurale.4373

ISSN: 2105-2581

\section{Publisher}

Société Française d'Économie Rurale (SFER)

\section{Printed version}

Date of publication: 15 July 2014

Number of pages: 87-106

ISSN: 0013-0559

\section{Electronic reference}

Sonia Bellit et Cécile Détang-Dessendre, «Les salariés agricoles. Entre ancrage sectoriel et précarité », Économie rurale [En ligne], 342 I juillet-août 2014, mis en ligne le 15 juillet 2016, consulté le 01 mai 2019. URL : http://journals.openedition.org/economierurale/4373 ; DOI : 10.4000/ economierurale.4373 


\title{
Les salariés agricoles Entre ancrage sectoriel et précarité
}

\author{
Sonia BELLIT • UMR 1041-Unité CESAER, INRA, Dijon; CCMSA, Paris \\ sonia.bellit@dijon.inra.fr \\ Cécile DÉTANG-DESSENDRE • UMR 1041-Unité CESAER, INRA, Dijon \\ Cecile.Detang-Dessendre@enesad.inra.fr
}

Les différents parcours professionnels des salariés travaillant dans les exploitations agricoles sont caractérisés et quelques clés de lectures, pour mieux les appréhender, sont proposées à partir d'une base de données originale, issue de la Mutualité sociale agricole (MSA). Une méthode d'analyse séquentielle, combinée avec une classification ascendante conduit à distinguer sept trajectoires types. Une première qualification de ces trajectoires permet de montrer comment celles-ci suivent pour partie des schémas observés dans les autres secteurs, notamment dans l'industrie, en se singularisant pour une autre partie du fait de certaines caractéristiques des emplois offerts par le secteur agricole, avec notamment des parcours de "saisonniers permanents ".

MOTS-CLÉS : trajectoires professionnelles, agriculture, Optimal Matching Analysis, classification, logit.

\section{Agricultural employees: between sectoral attachment and precarity}

The different career paths for employees working on farms are characterized and some keys to understand are produced from a original database, provided by the Mutualité sociale Agricole (MSA). We use the method of sequential analysis, combined with an upward classification led to distinguish seven different trajectories. A first qualification of these trajectories shows how they partly follow patterns observed in other sectors, especially industry, while singling out due to certain characteristics of the jobs offered by the agricultural sector, including "permanent seasonal courses." (JEL: J43, J64.)

KEYWORDS: Career path, agriculture, Optimal Matching Analysis, classification, logit.

D epuis près de 60 ans, nous assistons à de profondes mutations structurelles dans le secteur agricole. Ces changements ont impulsé en grande partie un phénomène récent : la salarisation de la maind'œuvre agricole. Entre 1988 et 2007, la part du travail familial, salarié ou non, sur l'ensemble des exploitations passe de $82 \%$ à $70 \%$. Le travail salarié (familial ou non) quant à lui représente $32,5 \%$ des Unités de travail annuel (UTA) ${ }^{1}$ en 2007 contre seulement $19 \%$ en 1988 (Cahuzac,

1. UTA : Unité de mesure de la quantité de travail humain sur une exploitation agricole. Une UTA équivaut à la quantité de travail annuel d'une personne à temps plein.
Détang-Dessendre, 2011). Ainsi, la diminution du nombre d'actifs (familiaux et salariés) s'est accompagnée d'une réorganisation de la main-d'œuvre agricole, le travail salarié se substituant progressivement au travail familial, même si le travail en agriculture reste pour partie une affaire de famille (Hervieu, Purseigle, 2013). En effet, cette salarisation passe aussi par l'adoption du statut de salarié d'un nombre croissant d'actifs familiaux, le nombre de salariés issus de famille agricole augmentant de $36 \%$ sur la même période. Blanc et al. (2008) mettent en évidence les liens existant entre la demande de travail salarié non familial et l'offre de travail familial émanant souvent du conjoint, 
des enfants et des parents de l'exploitant. L'augmentation de la première s'explique ainsi en partie par la réduction de moitié du temps de travail fourni par les seconds : les enfants sont scolarisés plus longtemps tandis que les conjoints ont des opportunités d'emplois en dehors de l'exploitation.

Historiquement, le salarié agricole était considéré comme un «paysan sans terre » sans qualités ni compétences reconnues. L'absence de terres était véritablement le seul attribut qui le dissociait de l'agriculteur. Il était donc partagé entre l'identité paysanne et ouvrière (Pharo et al., 1981). Aujourd'hui, le salariat agricole en France est reconnu, mais reste remarquable dans ses caractéristiques lorsqu'on le compare au salariat des autres secteurs économiques (Cahuzac, Détang-Dessendre, 2011) : très largement masculin (70\%), il est plus jeune en moyenne, peu qualifié (surreprésentation des salariés sans formation, $43 \%$ avec au mieux le brevet des collèges), relativement plus souvent à contrat à durée déterminée et mal rémunéré (rémunération en moyenne $20 \%$ inférieure à celle perçue par un ouvrier de l'industrie).

Cependant, on sait peu de choses sur les parcours professionnels de ces salariés, alors que différents travaux se sont intéressés au parcours des agriculteurs, de leur conjoint ou de leurs enfants (Errington, Gasson, 1994 ; Wallace et al., 1994). En mobilisant la théorie de la recherche d'emploi (Lippman, McCall, 1976) et les développements des modèles d'appariement (Jovanovic, 1979), ce travail empirique vise d'une part à mettre en évidence les différents parcours possibles et d'autre part, à apporter quelques clés de lectures pour mieux les appréhender. Nous avançons notamment des pistes pour distinguer les parcours selon leur niveau de stabilité dans le secteur agricole et plus généralement en emploi. Ce travail s'inscrit dans la réflexion sur la complexification généralisée des itinéraires professionnels (Davy, 2012), dans un contexte où des situations très diverses se construisent, particulièrement parmi les salariés les moins qualifiés, depuis des cas de disqualification sociale jusqu'à des parcours stabilisés en emplois à durée indéterminée (Palheta, Gehin, 2012).

Nous mobilisons dans ce travail une base de données originale, issue de la Mutualité sociale agricole (MSA). Nous avons reconstitué les trajectoires professionnelles, suites de situations professionnelles ordonnées dans le temps, avec un pas de temps de 15 jours, entre 2002 et 2005, d'un échantillon de 1312 individus ayant travaillé, en tant que salarié, au moins une fois dans une exploitation agricole, en caractérisant les périodes dans le secteur agricole, hors du secteur et les périodes de chômage. La caractérisation des trajectoires professionnelles, effectuée par une méthode d'analyse séquentielle combinée avec une classification ascendante hiérarchique (Confais, Nakache, 2000), conduit à distinguer sept trajectoires types. Elles se distinguent entre elles autant par leur niveau de stabilité que par leur niveau d'ancrage dans le secteur agricole. Nous proposons ensuite une première qualification de ces trajectoires types, précisant les caractéristiques des salariés et des marchés locaux du travail les plus représentés dans les différentes trajectoires. Pour cela, nous estimons un logit multinomial après s'être assuré que l'hypothèse d'indépendance des alternatives non pertinentes est acceptable.

Après avoir présenté dans une première section les hypothèses que nous formulons pour expliquer les trajectoires professionnelles des salariés en agriculture, nous présentons ensuite les données mobilisées pour mener à bien les tests empiriques. La troisième section est consacrée à la présentation de la typologie et la quatrième à l'analyse des déterminants des grands types de trajectoires. Une dernière section conclut ce travail. 


\section{Comment comprendre les parcours professionnels en agriculture?}

Les parcours professionnels sont le résultat de stratégies des salariés, conduites sous contrainte des stratégies des firmes et des conditions du marché. Après avoir rappelé les principaux résultats de la littérature sur les mécanismes qui régissent les parcours professionnels des salariés, nous nous appuyons sur les rares travaux qui, à notre connaissance, s'intéressent aux salariés agricoles pour avancer quelques hypothèses sur les spécificités du secteur.

Dans le cadre du modèle de recherche d'emploi de Lippman et McCall (1976), un individu en recherche d'emploi accepte un emploi dès lors que celui-ci lui offre un salaire supérieur à son salaire de réservation. Ce dernier égalise les coûts et les gains marginaux que représente la recherche d'emploi. Il dépend en général du revenu de substitution au travail, de la distribution des salaires dans l'économie et du taux d'arrivée des offres d'emploi, le dernier point étant source de friction. Le salaire est donc ici un déterminant important dans la décision d'accepter ou non une offre d'emploi $^{2}$. Si, dans le modèle de base, seuls les chômeurs recherchent un emploi, les travaux initiés par Van den Berg (Van den Berg, 1992 ; Van den Berg et al., 2002), qui, à la suite de ceux de Burdett (1978), intègrent la possibilité pour un salarié de rechercher un emploi plus satisfaisant tout en étant déjà en emploi, permettent d'étudier des successions d'emplois avec ou sans passage par le chômage.

L'impact négatif du temps passé au chômage sur la construction des parcours professionnels est bien documenté (Mincer, Ofek, 1982 ; Narendranathan, Elias, 1993). Jovanovic (1984) propose une autre hypothèse. Un salarié peut choisir de quitter un

2. Voir notamment Rogerson et al. (2005) pour une synthèse des différents modèles de recherche d'emploi. emploi pour le chômage si sa probabilité de recevoir une meilleure offre d'emploi est supérieure en étant au chômage qu'en emploi, la recherche étant plus efficace dans le premier cas de figure. Pour autant, l'impact négatif du chômage sur la stabilisation est empiriquement validé (Gagliarducci, 2005).

Certains développements se sont intéressés au rôle des contrats temporaires dans les parcours professionnels. Il s'agit en particulier d'évaluer dans quelles mesures le contrat temporaire permet d'accéder à un emploi stable et ainsi jouer le rôle d' " emploi pied à l'étrier », ou si au contraire ce type d'emploi est un frein à la stabilité. Les résultats empiriques sont mitigés. De Graaf-Zijl et al. (2011) montrent sur données néerlandaises que les emplois temporaires diminuent le temps passé au chômage. Pour Van Ours (2004), la succession d'emplois temporaires est un frein à l'accès à un emploi permanent : ce n'est pas tant la nature du contrat qui pose problème, mais la discontinuité qu'il suppose. Ainsi, la stabilisation du salarié est plus difficile lorsqu'il connaît des interruptions d'emploi (Gagliarducci, 2005).

Dans les travaux précités, le salaire est défini dès la formation de l'appariement salarié-poste. Autrement dit, au moment de l'accord entre le travailleur et l'entreprise, chacun dispose de toute l'information nécessaire pour fixer le salaire à la prise de fonction. Dans le cas des modèles d'appariement, les aptitudes du salarié ne sont pas révélées ex ante. La détermination du salaire est donc endogène et dépend de l'espérance de la qualité de l'appariement. Il est ensuite réévalué à chaque période et s'ajuste pour tendre vers la « vraie » productivité du travailleur (Jovanovic, 1979). Dans ce cadre, Boockmann et Hagen (2008) présentent le contrat à durée déterminée (CDD) comme le moyen de révéler à l'employeur la qualité de l'appariement à moindre coût. Les contrats temporaires 
offriraient la possibilité à l'employeur d'éliminer les mauvais appariements sans coûts de licenciement et de conserver uniquement les travailleurs compétents. Les auteurs valident empiriquement cette hypothèse. Ils constatent qu'après un certain temps, les périodes d'emplois débutées par un CDD se terminent moins fréquemment que celles initiées par un contrat permanent.

Le rôle du territoire sur les marchés du travail a longtemps été ignoré dans la modélisation, même si les travaux empiriques le mettent clairement en évidence. Partant du modèle de Harris et Todaro (1970), Zenou (2009) propose une formalisation des équilibres de marché, en tenant compte des différences entre espaces ruraux et urbains : salaires et taux d'offre d'emploi plus élevés dans l'urbain, mais aussi emplois moins qualifiés et taux de chômage plus faibles dans le rural (notamment du fait des frictions moindres dans la procédure de recherche). Selon Thisse et Zenou (1995), l'appariement entre travailleurs et entreprises est en moyenne meilleur dans un grand marché. Gaigné (2000) justifie les différences de salaire entre centres urbains et périphérie, pour un même type d'emploi, par la qualité de l'appariement plus faible en milieu rural. Par ailleurs, la probabilité de trouver une offre d'emploi acceptable à moindre coût est plus faible dans les zones rurales : d'une part, la demande de travail est dispersée et les opportunités sont donc moins nombreuses, d'autre part, la distance entre le lieu de résidence d'un travailleur rural et les centres d'emplois constituent des coûts de migrations élevés. Empiriquement, l'auteur met en évidence des relations d'emplois stables dans les espaces ruraux. Bien qu'ils perçoivent des salaires relativement faibles, l'éloignement des centres d'emplois urbains et la faible dotation en emplois freinent la mobilité professionnelle.

Gullstrand et Tezic (2008) étudient les sorties des salariés du secteur agricole vers d'autres secteurs en Suède. Les sorties du secteur agricole s'expliquent par (i) le niveau de salaire mesurant la qualité de l'appariement salarié-poste, (ii) les coûts de changement de poste et (iii) les coûts de changement de secteur. Les coûts de changement (de poste et de secteur) sont positivement liés au capital humain spécifique au poste et/ou au secteur. Bojnec et Dries (2005) s'intéressent aux causes des flux de travailleurs en provenance ou vers le secteur de l'agriculture. Le capital humain apparaît comme un déterminant majeur des mouvements intersectoriels. Les individus les plus éduqués sont plus susceptibles d'aller vers d'autres secteurs. À l'inverse, les individus les moins éduqués ont moins de chances de retrouver un emploi en dehors de l'agriculture et sont ainsi plus susceptibles d'y rester.

Pour étudier les différents parcours professionnels rencontrés dans la population des salariés ayant au moins une fois travaillé dans le secteur agricole, nous distinguons donc (i) les périodes d'emploi des périodes de chômage, (ii) les emplois stables des emplois à durée déterminée, les CDD non saisonniers (que l'on appellera CDD) des emplois saisonniers et enfin, (iii) les emplois dans le secteur agricole des emplois hors de ce secteur. Nous rapprochons ensuite les parcours, ainsi mis en évidence, des différents mécanismes précisés ci-dessus.

\section{Les données}

Pour reconstituer les trajectoires professionnelles, nous avons mobilisé des informations émanant de différentes sources, mises à disposition par la MSA. Une première base regroupe tous les contrats de travail actifs entre 2002 et 2005 des salariés cotisants au régime agricole. Elle met à notre disposition un éventail d'informations assez large sur l'emploi occupé lorsqu'il ouvre des droits au régime agricole (secteur d'activité, rémunération, 
date de début et de fin d'activité, type de contrat, etc.). À propos du salarié luimême, nous connaissons son sexe, son âge, sa nationalité et son lieu de résidence. Son niveau d'éducation, en revanche, est inconnu. Nous disposons également du recensement des chefs d'exploitation ${ }^{3}$ de la métropole cotisants à la MSA entre 2002 et 2005. Ce fichier fournit des informations sur l'exploitant lui-même (sexe, âge, situation familiale), le collectif de travail familial (conjoint, aide familial), les caractéristiques de l'exploitation (superficie, type d'exploitation, statut juridique, nature de l'activité et activité agro-touristique) et ses données économiques et financières (bénéfice, cotisations prélevées, salaires versés...). Nous utilisons donc ce second fichier pour relier le salarié agricole à l'exploitation dans laquelle il est employé, mais aussi retrouver les individus exerçant une activité au titre d'exploitant au cours de la période d'étude.

Les fichiers précédents ne renseignant que sur les périodes d'emploi couvertes par la MSA, nous avons eu recours à l'Échantillon inter-régimes des cotisants (EIC) pour compléter les trajectoires des salariés intégrant des périodes d'emploi hors de l'agriculture. Ce fichier suit un échantillon de cotisants aux différents régimes de retraite français. Nous travaillons sur l'échantillon de 2005, qui regroupe près de 240000 individus $^{4}$. Nous

3. Exploitant travaillant sur une exploitation de nature agricole ou connexe à l'agriculture.

4. L'EIC, élaboré par la Direction de la recherche, des études, de l'évaluation et des statistiques (DREES) est mis à jour tous les 4 ans. À chaque échéance, le fichier réunit tous les cotisants âgés de 31 ans à 71 ans. Le mode d'échantillonnage est ainsi glissant : chaque nouvel échantillon est composé des individus présents dans les EIC précédents (dont l'âge est inférieur ou égal à 71 ans) et d'un apport de cotisants âgés de 31 ans au moment de l'enquête. Le taux de sondage est d'une génération sur quatre entre 1934 et 1974 et de 2,68\% par génération. connaissons les dates de début et de fin d'activité (et non les durées effectives des contrats). Le type de contrat de travail (CDI, CDD, etc.) n'est en revanche pas précisé. Compte tenu des informations disponibles dans les différents fichiers, nous pouvons reconstituer les parcours professionnels de 6636 individus entre janvier 2002 et décembre 2005, soit quatre années.

Nous restreignons l'analyse aux individus ayant occupé un emploi dans la production (exploitations agricoles et entreprises de travaux agricoles [ETA]) au moins une fois durant leur parcours (3 602 individus). Nous éliminons également les salariés agricoles dont l'activité se limite exclusivement aux vendanges. En effet, les contrats vendanges sont destinés à un spectre relativement large de main-d'œuvre puisqu'ils peuvent être alimentés par des jeunes en cours d'études, mais aussi par des salariés en congés payés ou des fonctionnaires. Intégrer ces « vendangeurs exclusifs » dans l'analyse risquerait ainsi de masquer les régularités propres aux autres salariés agricoles : 785 individus ont été écartés. Nous centrons notre étude sur les individus ayant un contrat d'une durée minimum d'un mois ou plusieurs contrats d'une durée cumulée minimum de trois mois dans la production agricole. Cela revient à supprimer 1505 individus. Ce critère de sélection a pour objectif (i) de ne pas diluer les emplois saisonniers dans la masse de ces emplois de très courte durée, (ii) de focaliser l'analyse sur les salariés pour lesquels le secteur agricole constitue une étape dans le parcours professionnel. Il conduit donc à une sous-évaluation des parcours multisectoriels, pouvant alors donner à voir un secteur plus fermé qu'il ne l'est. Nous obtenons un échantillon final de 1312 individus. Différentes informations sur leur vie professionnelle avant 2002 sont disponibles, notamment leur expérience dans le secteur agricole. Cependant, nous sommes en présence de données censurées à gauche puisque certains épisodes ont débuté avant le $1^{\text {er }}$ janvier 2002. De la même 
façon, les épisodes encore en cours à la fin de la période d'étude, soit au 31 décembre 2005, sont censurés à droite. Ces censures amènent à une sous-évaluation de la durée des épisodes.

Nous retrouvons dans notre échantillon les traits caractéristiques de la maind'œuvre agricole décrits dans Cahuzac et Détang-Dessendre (2011) : majoritairement des hommes (60\%), légèrement plus jeunes que la moyenne de la population active (39 ans) (voir annexe). La répartition des types de contrat des 568 salariés en emploi dans le secteur de la production agricole en 2005, est aussi très comparable à ce que les auteurs observent dans l'Enquête emploi (EE) : 74,1\% sont en CDI dans notre échantillon contre $74,3 \%$ dans EE, $11,3 \%$ en CDD non saisonnier contre $12,2 \%$ dans EE et $14,6 \%$ en emploi saisonnier contre $14,6 \%$ dans EE. La part des CDI reste assez stable entre 2002 et 2005. Quant à la proportion des CDD agricoles non saisonniers et des contrats courts non agricoles, elle diminue de 1,5 point de pourcentage seulement. Les chômeurs, quant à eux, représentent un tiers de l'échantillon au début et à la fin de la période d'étude, marquant les difficultés d'insertion rencontrées par les salariés passant dans le secteur.

Ces constats généraux masquent néanmoins les transitions éventuelles entre les différentes situations possibles. Ainsi, nous avons étudié les situations observées en 2005 conditionnellement aux situations initiales de 2002. Parmi les salariés agricoles en CDI en 2002, plus des deux tiers sont dans la même situation en 2005 et $12 \%$ sont au chômage, soit une proportion à peine supérieure à la moyenne nationale. Si l'on ajoute ceux qui occupent un emploi stable hors agriculture en 2005, se dessine donc une catégorie de salariés pour lesquels la situation sur le marché du travail est stable. Concernant les saisonniers de notre échantillon en 2002, la moitié est au chômage en 2005 et $20 \%$ environ occupent encore un emploi temporaire. En revanche, les titulaires d'un CDD agricole non saisonnier semblent accéder plus facilement à un emploi stable : un peu plus d'un tiers d'entre eux sont en CDI agricole ou en contrat long hors agriculture en 2005. Ce chiffre est à relativiser puisqu'un autre tiers est en situation de chômage à la fin de la période. Des salariés cumulant emploi en agriculture et hors agriculture en 2002 (40\%) occupent un emploi stable en agriculture $(30 \%)$ ou hors agriculture $(10 \%)$ en 2005. Les chômeurs, quant à eux, ont un taux d'accès à l'emploi stable relativement proche des titulaires d'un contrat saisonnier en 2002. En effet, seulement 19 \% des individus au chômage en 2002 occupent un emploi stable en 2005 et $22 \%$ ont un emploi temporaire. Les épisodes de chômage semblent être une trappe à la précarité puisque $57 \%$ d'entre eux sont encore en situation de chômage en 2005.

\section{Construction d'une typologie des trajectoires des salariés de la production agricole}

Définissant la trajectoire comme une suite de situations professionnelles ordonnée dans le temps, il s'agit désormais de synthétiser l'information. L'étude et la comparaison des trajectoires professionnelles supposent en effet une nomenclature d'états possibles relativement restreinte. Nous distinguons tout d'abord les contrats du secteur agricole des contrats hors de l'agriculture. Dans le cas des emplois hors de l'agriculture, nous sommes amenés à considérer les emplois de moins d'un an comme des « emplois courts » et les autres comme des « emplois longs $»^{5}$. Dans le cas des emplois $\mathrm{du}$ secteur agricole, nous dissocions les contrats saisonniers des autres CDD.

5. Cette simplification a probablement pour conséquence une surestimation des « contrats courts » hors de l'agriculture puisque tout CDI débutant en cours d'année sera par construction considéré comme un contrat de courte durée. 
En construisant une typologie de trajectoires professionnelles, nous choisissons d'adopter dans un premier temps une approche descriptive. En pratique, la démarche typologique consiste tout d'abord à déterminer une mesure de distance entre les trajectoires. À partir de la matrice de distance, nous pouvons ensuite déterminer les trajectoires analogues ou au contraire dissemblables au moyen d'une méthode de classification. Afin de calculer la distance entre les différentes trajectoires, nous optons pour une méthode d'analyse séquentielle : l'Optimal Matching Analysis (OMA), méthode développée à l'origine en biologie moléculaire pour analyser les séquences d'ADN. Le principe de l'OMA est d'estimer la similarité/dissimilarité entre deux trajectoires en calculant le coût représenté par la transformation d'une séquence, suite d'événements ordonnés qui compose le parcours professionnel, en une autre (voir encadré méthodologique) (Robette, 2011).

\section{Encadré méthodologique. Distance entre trajectoires et construction de la typologie}

La distance entre deux séquences correspond au coût minimal nécessaire à la transformation d'une séquence en l'autre. Ce coût dépend de trois opérations : la suppression ou l'insertion d'un événement de la trajectoire ou la substitution d'un événement par un autre. Les opérations de suppression/insertion, considérées comme équivalentes, privilégient l'ordre des événements, mais modifient la structure temporelle des séquences. À l'inverse, les opérations de substitution bouleversent l'enchaînement des événements tout en conservant leur structure temporelle. Faut-il alors considérer comme étant similaires deux trajectoires ayant des situations identiques au même moment d'observation (intérêt porté à la structure temporelle des événements) ou deux trajectoires ayant des transitions identiques à différents moments d'observations (intérêt porté à la nature des transitions) ? En pratique, le coût de substitution est calculé à partir des probabilités de transitions entre les états. Par exemple, les transitions entre les états « chômage » et « saisonnier » étant relativement plus fréquentes que celles entre « chômage » et « CDI », le coût de substitution entre « chômage » et « saisonnier » est ainsi moins élevé $(1,978)$ que celui entre « chômage » et «CDI » $(1,996)$. La probabilité de transition de l'état $C_{i}$ à l'état $\mathrm{C}_{j}$ est :

$$
P\left(C_{i} / C_{j}\right)=\frac{\sum_{t=1}^{L-1} n_{t, t+1}\left(C_{i}, C_{j}\right)}{\sum_{t=1}^{L-1} n_{t}\left(C_{i}\right)}
$$

avec $\mathrm{L}$ la longueur maximale des séquences observées, $n_{t}\left(\mathrm{C}_{i}\right)$ le nombre de séquences avec l'état $\mathrm{C}_{i}$ à la position $t \in\{1 \ldots L-1\}$, $\mathrm{n}_{t, t+1}\left(\mathrm{C}_{i}, \mathrm{C}_{j}\right)$, le nombre de séquences avec l'état $\mathrm{C}_{i}$ à la position $t$ et l'état $\mathrm{C}_{j}$ à la position $t+1$. Un coût de substitution de référence entre deux états est alors égal à 2 auquel on soustrait la probabilité de transition (Rohwer, Pötter, 2005). Par conséquent, plus la probabilité de transition entre deux états est élevée, plus leur coût de substitution sera faible. Nous fixons enfin les coûts d'insertion et suppression à la moitié du coût de substitution minimal en prenant ainsi en compte parallèlement la simultanéité des événements et leur ordre. (Robette, 2011).

À partir de la matrice de distance entre les séquences, nous appliquons la méthode de classification ascendante hiérarchique par le critère de Ward (Confais, Nakache, 2000). Elle consiste à regrouper de manière itérative les parcours qui se ressemblent le plus tout en veillant à minimiser l'hétérogénéité intra classe et au contraire à maximiser l'hétérogénéité inter classe à chaque étape. Nous pouvons dès lors déterminer le nombre de classes de la typologie à partir d'indicateurs statistiques tels que le saut d'inertie. Lorsque la différence d'inertie entre deux niveaux de partitions voisins est faible, l'augmentation du nombre de classes apporte un supplément d'information négligeable. L'étude des sauts d'inertie entre les différents niveaux de partition des données (figure 1) nous amène à retenir une typologie en sept classes. 
Figure 1. Inertie de la partition selon le nombre de classes

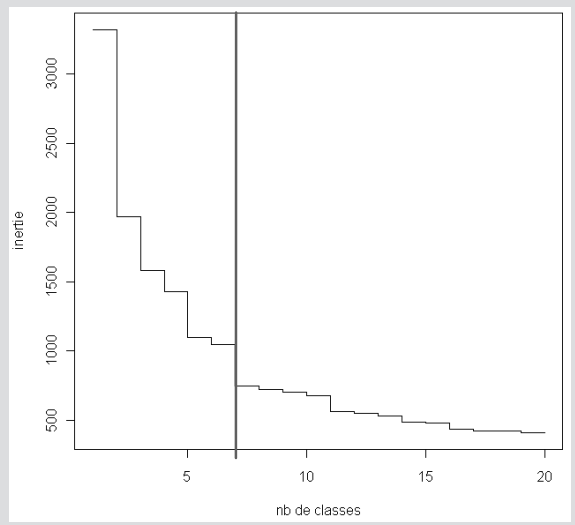

Afin de décrire les différents types de parcours professionnels, nous nous appuyons sur les graphiques des " tapis de trajectoires », divers indicateurs synthétiques et les «parangons » de chaque classe.

En représentant une ligne par trajectoire et une couleur différente à chaque changement de situation, le tapis de trajectoires permet de rendre compte des transitions éventuelles rencontrées par les individus d'une classe. Prenons deux exemples, la catégorie des « stables en agriculture » et celle des « chômeurs récurrents ».

Afin de décrire les différents types de parcours professionnels, nous nous appuyons sur les graphiques des « tapis de trajectoires », divers indicateurs synthétiques et les «parangons » de chaque classe.

En représentant une ligne par trajectoire et une couleur différente à chaque changement

\section{Figure E.1. Stable en agriculture 1}

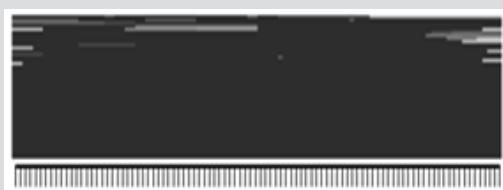

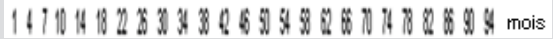

de situation, le tapis de trajectoires permet de rendre compte des transitions éventuelles rencontrées par les individus d'une classe. Prenons deux exemples, la catégorie des " stables en agriculture » et celle des « chômeurs récurrents ».

Dans la figure E.1, le poids important des CDI agricoles de longue durée est matérialisé par la dominance gris foncé, couleur représentant ce type d'épisode. Ce tapis de trajectoire uniforme illustre tant l'homogénéité des trajectoires de cette catégorie, que la durée des contrats. À l'opposé, le gris le plus clair, dominant dans la figure E.2 matérialise l'importance des périodes longues de chômage, entrecoupées par des périodes souvent courtes parmi lesquelles aucune couleur ne domine vraiment illustrant la grande diversité des périodes d'emploi connues.

Les parangons de chaque classe correspondent à la/les trajectoire(s) professionnelle(s) la plus proche du centre de gravité et complètent la caractérisation des trajectoires. Lorsqu'une classe ne peut pas être résumée par un seul modèle de trajectoire, nous nous référons à un groupe de trajectoires représentatives déterminées par un algorithme de recherche (Gabadinho et al., 2010) basé sur les densités de voisinage. À titre illustratif, deux trajectoires représentent la catégorie « $\mathrm{CDD}$ en agriculture » (figure E.3). La première correspond à une période en CDD agricole (gris medium) suivie d'une stabilisation (gris foncé), la deuxième correspond à une courte période de chômage (gris clair) suivie d'une période en CDD agricole (gris medium).

Figure E.2. Chômeurs récurrents

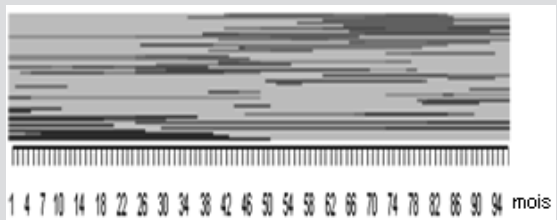

\section{Figure E.3. CDD en agriculture}

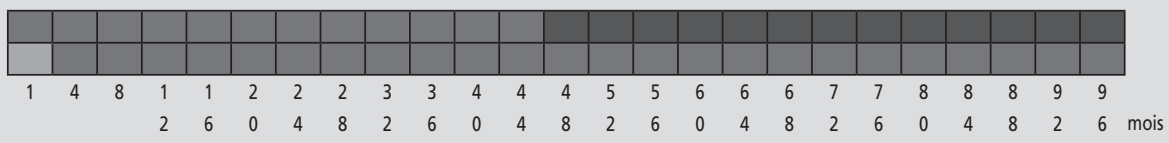


RECHERCHES

Sonia BELLIT, Cécile DÉTANG-DESSENDRE

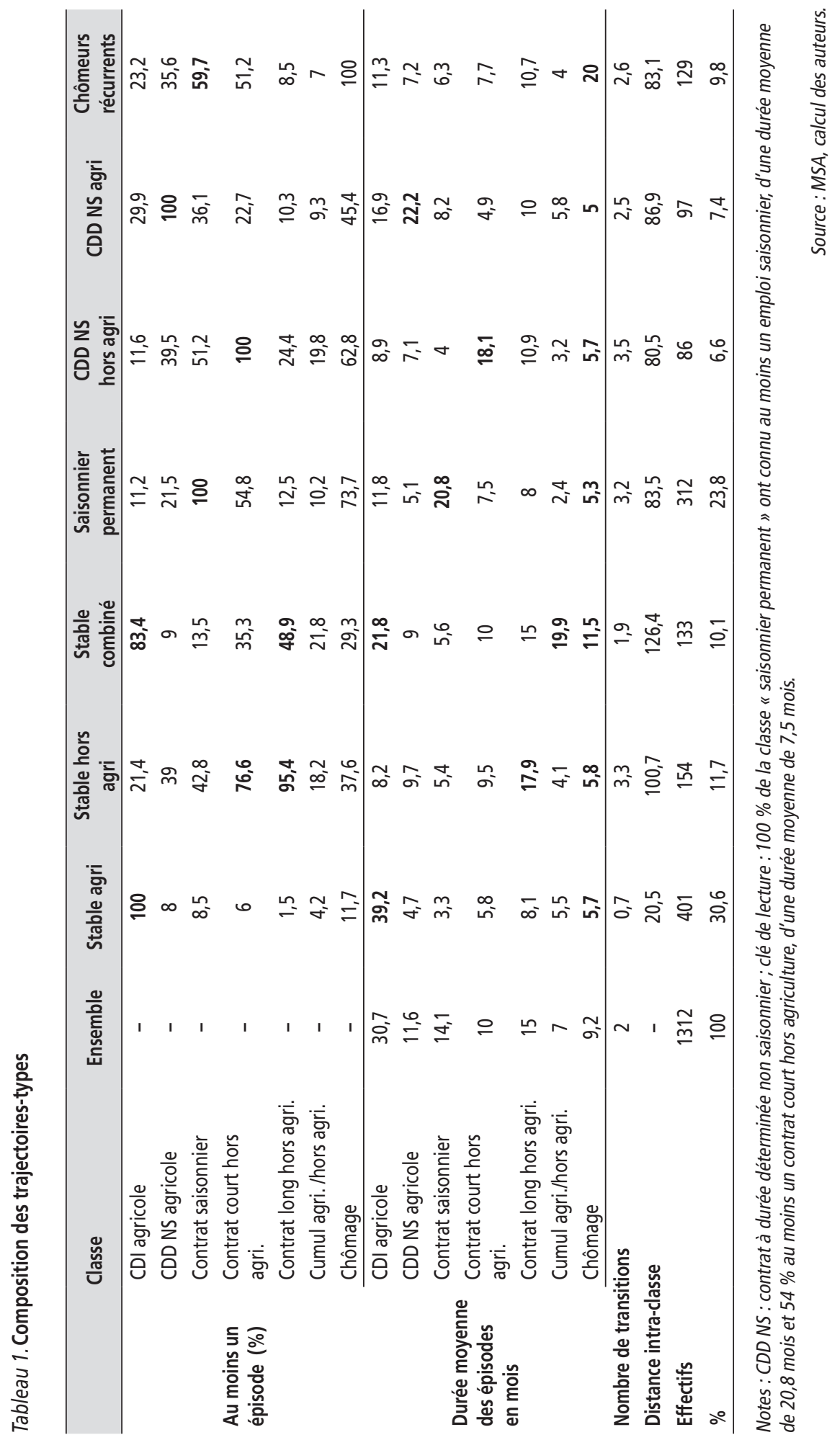




\section{Sept grands types de trajectoires}

La grande diversité des parcours professionnels des individus peu qualifiés décrite par Palheta et Gehin (2012) se retrouve dans notre échantillon de salariés.

Un peu plus de la moitié d'entre eux ont suivi au cours des 5 années observées une trajectoire qui présente des caractéristiques de stabilité : des emplois en CDI, sur une longue durée, avec des passages au chômage peu fréquents et plutôt brefs. Au sein de ce groupe se distinguent les parcours construits presque exclusivement dans le secteur agricole $(58 \%)$ des parcours où le secteur agricole se combine avec d'autres secteurs, pour n'être que très accessoire dans certains cas (tableau 1).

De l'autre côté de l'axe de stabilité ( $f$ gure 2) se déclinent des situations très différentes les unes des autres, mais toutes marquées par un certain niveau de précarité. Un premier type regroupe les parcours les plus précaires, avec des périodes de chômage longues et des passages par l'emploi, en agriculture ou dans un autre secteur, de courte durée. Cette catégorie représente $20 \%$ des parcours précaires (10\% de l'ensemble des parcours). Enfin, les trois types de parcours construits sur l'enchaînement d'emplois temporaires se distinguent par la place du secteur agricole et lorsque celui-ci est prépondérant, par le type de contrat dominant. Lorsque les emplois courts hors du secteur agricole sont majoritaires, ils sont souvent assez longs, alors que les passages par l'agriculture sont composés d'emplois de courte durée, les passages au chômage étant aussi brefs. Lorsque les emplois en contrat à durée déterminée dans le secteur agricole sont majoritaires, on observe l'image inversée : les expériences hors de l'agriculture sont brèves. Le passage au chômage est moins fréquent que dans la catégorie précédente et aussi court.

Figure 2. Les différents types de trajectoires

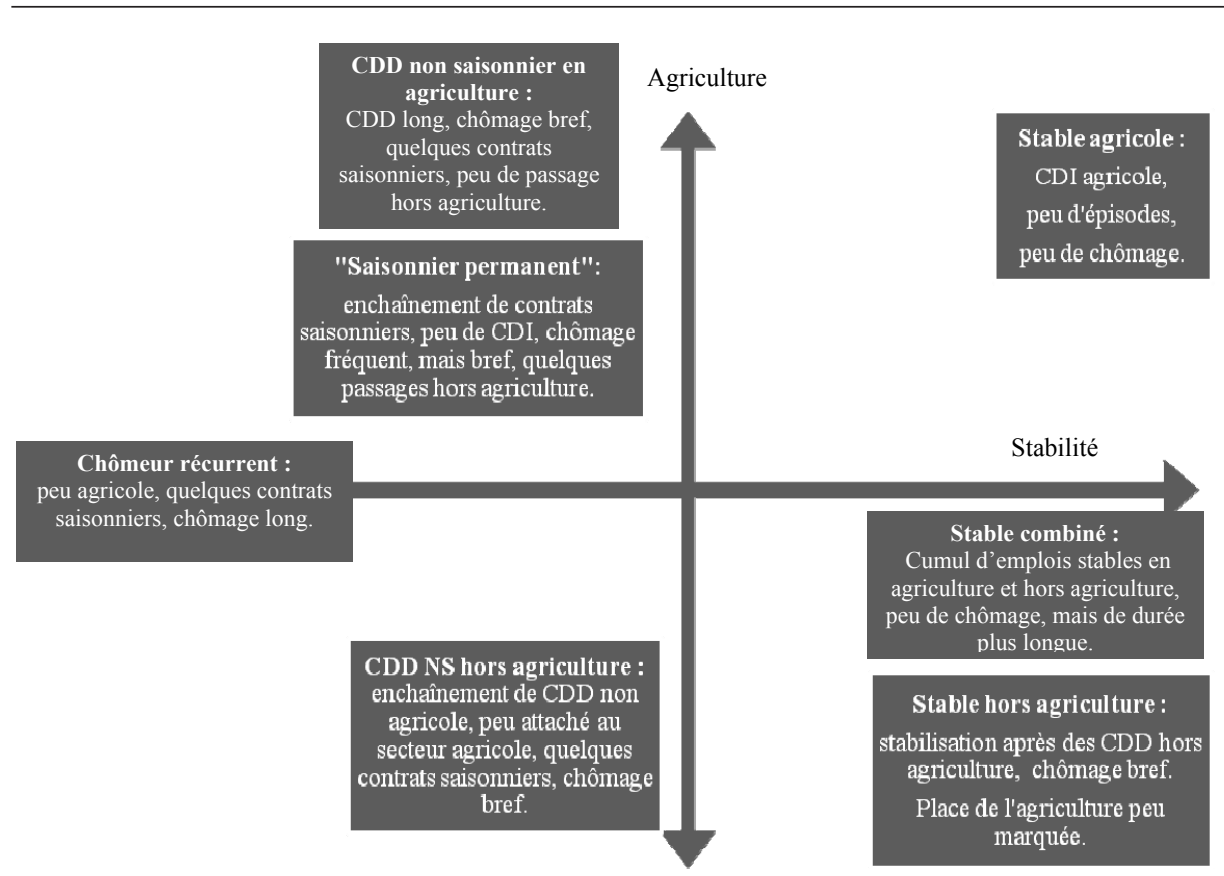

Source : les auteurs. 
Enfin, ce travail permet de mettre en lumière un type de trajectoire singulier, construit sur l'enchaînement d'emplois saisonniers, de longues durées (plus de 20 mois en moyenne), entrecoupés par des périodes, courtes, de chômage.

\section{Une stabilité professionnelle dans le secteur agricole est possible, mais non majoritaire}

Plus en détail, la classe marquée par des emplois stables en agriculture (CDI agricole) regroupe $30 \%$ des salariés de l'échantillon : $80 \%$ des contrats de cette classe sont des CDI agricoles en début et en fin de période et la majorité des salariés est en CDI durant toute la durée d'étude. La durée des épisodes en CDI agricole est en moyenne de 39 mois, soit un peu plus de 3 ans. Le parangon (voir encadré) est un homme de 32 ans en 2002 et de nationalité française. Il est en CDI durant toute la période et ne change pas de contrat. Il exerce le métier d'ouvrier agricole à temps plein sur une exploitation d'élevage porcin (voir annexe pour une description statistique).

Des salariés de l'échantillon (22\%) ont construit un parcours structuré par des emplois stables hors de l'agriculture. Pour les individus les plus éloignés du secteur, leur passage par le secteur agricole est rapide puisqu'ils y restent en moyenne sept mois $^{6}$. Les épisodes d'emploi en contrat long hors agriculture durent quant à eux en moyenne un an et demi. Le parcours-type correspond à un court passage en agriculture suivi d'une sortie du secteur par le biais d'un CDD et enfin d'une "stabilisation » professionnelle hors de l'agriculture. Ces trajectoires se stabilisent plus progressivement que celles des salariés stables en agriculture, les premières connaissant

6. Moyenne des durées moyennes des épisodes en CDI agricole, CDD agricole, contrat saisonnier agricole et en cumul agriculture/hors agriculture. moins d'une transition en moyenne sur les 5 ans, alors que les secondes plus de 3 .

Se présentant comme un intermédiaire entre les deux précédentes catégories, un groupe est constitué de salariés qui combinent des emplois en CDI dans le secteur agricole et hors de l'agriculture. Les salariés cumulant emplois en agriculture et hors agriculture sur toute la période sont aussi représentés. Par ailleurs, ces périodes de cumul d'emplois sont relativement longues : elles durent en moyenne un an et demi. De manière générale, la plupart des individus alternent entre situations permanentes en agriculture et hors de l'agriculture (souvent entrecoupées par un ou plusieurs contrats courts non agricoles) ou occupent simultanément des emplois dans les deux domaines d'activité. Ces parcours sont marqués par leur ouverture vers d'autres secteurs que l'agriculture.

\section{La nature des contrats distingue les trajectoires accumulant des emplois temporaires}

Trois classes de trajectoires sont particulièrement marquées par la récurrence de contrats courts, se distinguant alors par le niveau d'ancrage des emplois dans le secteur agricole. Parmi les trajectoires marquées par ce secteur, une distinction s'effectue entre les types de contrats euxmêmes, entre CDD non saisonnier et emplois saisonniers. Ce travail de typologie met en lumière une forme de tri entre les salariés agricoles. On ne combine en effet que rarement emplois saisonniers et autres CDD. Plus précisément, les 312 individus de la classe des saisonniers récurrents, soit près de $24 \%$ de l'échantillon, enchaînent des contrats saisonniers agricoles entrecoupés de courtes périodes de chômage. Les épisodes d'emploi en contrat saisonnier durent un peu moins de deux ans en moyenne. Paradoxalement, au caractère temporaire des contrats saisonniers 
s'oppose ici la notion de persistance. Le parangon est un homme d'origine étrangère dont la trajectoire se résume à un enchaînement de contrats saisonniers entre deux courtes périodes de chômage. Durant quatre ans, cet homme a exercé son métier au sein de huit exploitations différentes, spécialisées dans des activités de viticulture, de cultures de céréales ou de cultures fruitières. Le salarié est relativement mobile puisqu'il travaille dans plusieurs départements du sud-est de la France, à savoir les Hautes-Alpes, la Drôme et le Gard.

La majorité des individus en CDD agricole sur une longue période a des contrats de près de deux ans en moyenne. Dans cette catégorie, deux grands types se dessinent ; ceux qui enchaînent les contrats courts, et ceux qui accèdent à un CDI. La première trajectoire représentative est celle d'une femme française de 36 ans. Elle cumule trois CDD consécutifs dans une unique exploitation de culture de légumes entre 2002 et juillet 2005. Elle se retrouve ensuite en situation de chômage. La seconde trajectoire représentative est celle d'un homme français de 32 ans. Après un CDD à temps plein de deux ans sur une exploitation de services aux cultures productives, celle-ci transforme son contrat en CDI. Au-delà de la non-porosité entre emplois en CDD et emplois saisonniers, cette première description laisse aussi entrevoir des parcours se différenciant quant à leur issue. En effet, dans la classe regroupant les parcours marqués par les CDD, $30 \%$ accèdent à la stabilisation.

Lorsque les contrats courts hors de l'agriculture constituent l'essentiel des épisodes de la trajectoire des salariés, les passages en agriculture sont aussi sous statut temporaire (CDD ou saisonnier) et de courte durée. Cette catégorie est la plus instable et les salariés connaissent en moyenne 3,5 transitions contre seulement 2 transitions pour l'ensemble de l'échantillon. La plupart des trajectoires correspondent à de longues périodes d'enchaînements d'emplois courts en dehors de l'agriculture précédées et suivies de périodes de chômages et/ou de contrats courts agricoles. Les passages en agriculture ne sont pas durables et ne constituent pas en cela un véritable ancrage dans le secteur. Ils sont plutôt le moyen pour les individus de rester en emploi.

\section{Des trajectoires chaotiques où le passage par l'agriculture n'est pas significatif}

Une très longue période de chômage (en moyenne près de 2 ans) entrecoupée de contrats temporaires en agriculture ou hors de l'agriculture caractérise les parcours du dernier type de trajectoire. Les passages en emploi sont relativement courts. Les contrats temporaires (contrats en agriculture et hors de l'agriculture confondus) durent en moyenne deux à cinq fois moins longtemps que les périodes de chômage. Ces individus ne parviennent donc visiblement pas à s'insérer durablement sur le marché du travail. Dès lors, les contrats courts en agriculture apparaissent comme un moyen de reprendre occasionnellement une activité. Le parangon de cette catégorie est un homme français de 28 ans. Il connaît une période de chômage de trois ans et demi suivie d'une période d'emploi saisonnier en agriculture de deux mois et demi puis d'un retour au chômage en fin 2005. Cet individu réside en Charente, département réputé principalement pour ses activités de viticulture.

\section{1. Éléments de qualification des trajectoires professionnelles en agriculture}

Nous cherchons ensuite à tester les hypothèses quant aux déterminants des différents types de trajectoires possibles dans le secteur agricole. Il s'agit en particulier de mettre en relation le type de trajectoire suivi par le salarié et ses caractéristiques 
d'une part, et celles de son environnement local d'autre part.

Les hypothèses que nous souhaitons tester sont les suivantes :

- Les trajectoires stables (hors ou en agriculture), avec peu de transitions, devraient concerner plus particulièrement les salariés les plus qualifiés, la qualité de l'appariement étant telle que le salarié ne peut guère espérer une amélioration significative de son salaire (Jovanovic, 1979).

- Si on retient l'hypothèse selon laquelle les emplois saisonniers sont essentiellement des emplois de conditionnement, de cueillette, très peu qualifiés, alors les parcours marqués par ce type d'emploi devraient se distinguer par une forte instabilité, touchant plus particulièrement les femmes et les salariés les moins qualifiés.

- Resteront dans le secteur agricole ceux qui auront le capital humain spécifique (au secteur) le plus important (Gullstrand, Tezic, 2008).

- La probabilité de trouver un emploi long est aussi conditionnée par le taux d'offres d'emploi qui arrivent aux salariés. Les salariés localisés dans les espaces les plus éloignés des centres d'emploi devraient avoir un taux d'arrivée d'offres faible (Gaigné, 2000 ; Thisse, Zenou, 1995), conduisant à une plus grande stabilité en emploi, même avec un salaire faible. De plus, un taux de chômage local plus élevé devrait augmenter la probabilité de cumuler les emplois courts, ainsi que les périodes de chômage.

- La stabilisation dans l'agriculture dépendra des offres d'emploi du secteur accessibles aux salariés, et notamment l'orientation productive de la zone, mais aussi de la concurrence entre l'agriculture et les autres secteurs. Si les offres des autres secteurs sont importantes, le salarié peut être amené à quitter le secteur agricole. Les employeurs peuvent alors réagir en offrant plus d'emplois stables.

Le modèle logit multinomial est un outil qui permet de relier la probabilité de réalisation d'un évènement à un ensemble de variables explicatives (Thomas, 2000). Ce modèle repose sur l'hypothèse d'indépendance des alternatives non pertinentes qui implique dans notre cas que la probabilité pour un individu de suivre un type de parcours plutôt qu'un autre est indépendante des autres parcours possibles. Nous testons cette hypothèse en mettant en œuvre tout d'abord le test de Hausman et McFadden (1984), puis la spécification proposée par Small et Hsiao (1985), basée sur la différence des log-vraisemblances. Le test nous permet d'accepter l'hypothèse d'indépendance et donc d'utiliser cette modélisation pour une première analyse des parcours mis en évidence?

La catégorie stable en agriculture est retenue comme référence. Pour tester l'impact relatif des caractéristiques individuelles (hypothèses 1 à 3 ) et des caractéristiques du marché local du travail (hypothèses 4 et 5) sur les trajectoires des salariés, les variables suivantes sont utilisées : (i) le sexe, l'âge, la nationalité, la profession, la catégorie socioprofessionnelle (PCS) la plus élevée observée et l'expérience en agriculture avant 2002 pour caractériser l'individu ; (ii) la catégorie d'espace du lieu de travail sur un gradient urbain-rural et la distance au centre de plus de 50000 habitants $^{8}$ pour préciser

7. La valeur du test d'Hausman et McFadden étant négative pour quatre des six tests (nous éliminons chaque alternative successivement de 2 à 7 avec la classe 1 comme référence), nous utilisons le test de Small et Hsiao dont les valeurs sont : $\operatorname{elim} 2=0,406 ; \operatorname{elim} 3=0,311 ; \operatorname{elim} 4=0,832$; $\operatorname{elim} 5=0,531 ; \operatorname{elim} 6=0,867 ; \operatorname{elim} 7=0,444$. Nous ne rejetons pas l'hypothèse d'indépendance des alternatives non pertinentes.

8. La distance de centre à centre a été calculée à l'aide du logiciel Odomatrix. INRA UMR1041 Cesaer, d'après IGN route 500 . 
l'accessibilité aux emplois et la structure sectorielle, le type d'activité agricole et le taux de chômage du bassin de vie (Julien, Pougnard, 2004) pour qualifier le marché local du travail.

\section{Un secteur soumis aux mécanismes standards, mais avec quelques spécificités}

La première hypothèse testée, classique en économie du travail, concerne le rôle du niveau de qualification dans la construction des parcours professionnels. Ne disposant pas d'information sur le niveau de formation, un indicateur a été construit sur la base des informations disponibles sur les emplois occupés. Les salariés les plus qualifiés (ceux ayant au moins une fois occupé un emploi d'encadrement) ont une probabilité plus élevée que les autres de suivre des trajectoires conduisant à la stabilité professionnelle, dans le secteur agricole ou hors de l'agriculture (tableau 2). Ils sont en effet moins souvent sur des trajectoires marquées par des contrats courts ou par du chômage récurrent et de longue durée. En cela, le schéma suivi par cette population passant par l'agriculture ne se distingue pas de ceux observés sur l'ensemble des salariés.

Les salariés qui ont uniquement occupé des emplois d'ouvriers non qualifiés se distinguent des ouvriers qualifiés et employés par leurs parcours plus ancrés dans le secteur agricole (parcours stable ou succession de CDD, saisonniers ou non) que dans les autres secteurs. La différenciation sectorielle des offres est certainement en jeu dans ce phénomène. En effet, le faible niveau de qualification des emplois proposés par le secteur productif agricole permet d'offrir des parcours stables à des salariés sans qualification. On distingue les salariés de notre échantillon selon leur nationalité française ou étrangère. Des travaux montrent que les parcours professionnels sont plus souvent chaotiques et que la stabilisation est plus difficile pour les salariés d'origine étrangère (Battu, Zenou, 2010). Les résultats sont moins tranchés sur notre population d'étude. En effet, il semblerait que les salariés de nationalité étrangère sont plus souvent ancrés dans le secteur agricole, y compris sur des trajectoires stables.

L'hypothèse selon laquelle le niveau de capital humain spécifique dans un secteur augmenterait la probabilité de s'y stabiliser est confirmée dans le cas du secteur de la production agricole : un bon niveau de capital spécifique au secteur agricole acquis avant la période d'étude (expérience en agriculture) augmente la probabilité de poursuivre une trajectoire stable en agriculture.

La sur-représentation des femmes dans les parcours instables notée dans la description des classes, se retrouve dans l'analyse à toutes choses égales par ailleurs, lorsque l'on ne tient pas compte de l'expérience en agriculture acquise avant la période considérée 9 . Alors, les femmes ont une probabilité plus élevée d'enchaîner des emplois instables hors de l'agriculture ou dans le secteur agricole, et tout particulièrement dans des emplois saisonniers. En revanche, lorsque l'on contrôle l'expérience professionnelle en agriculture et le genre, la seule différence qui subsiste concerne les emplois saisonniers. Ainsi, lorsque les femmes s'ancrent dans le secteur agricole, leur probabilité de stabilisation ne semble pas très différente de celle des hommes. Reste que les emplois saisonniers sont plus souvent féminins.

9. Estimation disponible auprès des auteurs. 
RECHERCHES

Sonia BELLIT, Cécile DÉTANG-DESSENDRE

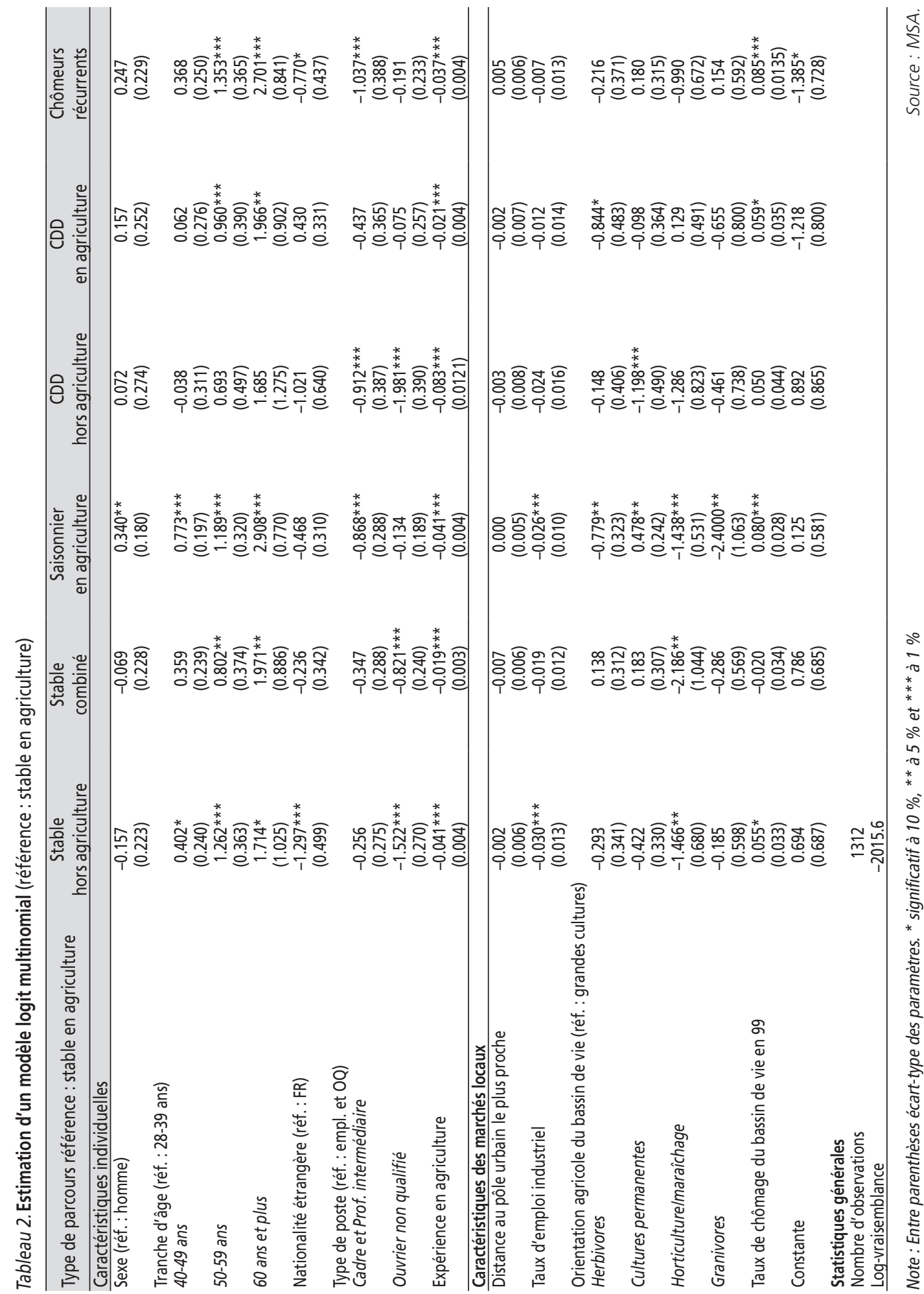




\section{Les conditions locales d'emploi conditionnent la nature des trajectoires professionnelles}

La densité et la nature des offres d'emploi qui arrivent aux salariés influencent leur trajectoire professionnelle. La densité de l'offre, plus faible lorsque l'on s'éloigne des centres urbains, a deux effets attendus : la faiblesse des offres conduit les salariés à rester dans un emploi qui ne les satisfait pas entièrement, et de ce fait augmente la stabilité ; elle peut aussi diminuer la probabilité d'accéder à un emploi et donc augmenter les trajectoires de grande précarité. Deux variables sont introduites: le niveau de chômage de la zone d'emploi et la distance à un pôle d'emploi. Les salariés résidant dans des zones d'emploi à fort taux de chômage suivent plus souvent des trajectoires précaires, avec des épisodes de chômage, nombreuses ou fortement marquées par l'emploi saisonnier. La distance au pôle urbain le plus proche, avec différents seuils de taille de pôle, a été introduite. Nous avons, en effet, testé (i) la distance au pôle urbain le plus proche (quelle que soit sa taille), (ii) la distance à un pôle de plus de 50000 habitants, (iii) la distance à un pôle de plus de 100000 habitants et (iv) la distance à un pôle de plus de 200000 habitants. Aucune distinction significative ne se dessine ni en matière de stabilité, ni en ce qui concerne la place du secteur agricole dans les parcours en fonction de la distance à un centre urbain, quel que soit le seuil de taille testé. Toutefois, il faut noter que l'essentiel de la population étudiée réside dans le rural ou dans des petites villes.

La structure des emplois offerts aux salariés devrait conditionner les parcours et en particulier leur niveau d'ancrage dans le secteur agricole. Le niveau de concurrence entre le secteur agricole et les autres secteurs peut avoir deux effets opposés, comme nous l'avons précisé précédemment : si les offres venant des secteurs non agricoles sont importantes, cela peut conduire le salarié à quitter le secteur. Inversement, ce risque peut alors conduire les employeurs agricoles à proposer plus d'emplois stables pour garder la maind'œuvre. Empiriquement, la probabilité d'avoir un parcours stable en agriculture (plutôt que stable hors de l'agriculture) augmente avec le taux d'emploi industriel. Ce résultat est à préciser, mais tout se passe comme si les employeurs agricoles adaptent leurs offres et cherchent à stabiliser la main-d'œuvre en agriculture lorsque la concurrence locale avec le secteur industriel est forte.

Enfin, l'orientation productive des zones d'emploi conditionne le type d'emploi proposé en agriculture (Peltier et al., 2009). Par exemple, les secteurs de la viticulture, de l'arboriculture, des grandes cultures et des cultures maraîchères, très marqués par la saisonnalité, emploient les trois quarts de la main-d'œuvre saisonnière ce qui devrait peser sur les trajectoires suivies dans les régions marquées par ces spécialisations. Ainsi, une orientation productive dans les cultures permanentes est, de façon attendue, liée positivement aux parcours caractérisés par l'enchaînement de contrats saisonniers. En revanche, une orientation productive en horticulture/maraîchage favorise les trajectoires stables en agriculture ou marquées par l'enchaînement de CDD agricoles.

$$
\begin{gathered}
* \\
* *
\end{gathered}
$$

Des mutations profondes des formes de travail dans le secteur de la production agricole sont en cours. En 2008, près de 230000 salariés travaillaient dans ce secteur. Pour comparaison, l'industrie automobile employait 330000 salariés cette même année. Pour autant, ce salariat agricole multiforme est peu étudié. Ce travail a pour principal objectif de présenter un état des lieux des trajectoires professionnelles des salariés agricoles. Une particularité du travail en agriculture est sa grande 
RECHERCHES

Sonia BELLIT, Cécile DÉTANG-DESSENDRE

saisonnalité, conduisant à une instabilité des statuts. Cependant, se dégagent de ce travail des trajectoires de « saisonniers permanents » dans le sens où ces salariés inscrivent leurs activités de saisonniers dans la durée. Six autres types de trajectoires se dessinent, combinant niveau d'ancrage dans le secteur agricole et niveau de stabilité.

Des mécanismes bien documentés sur l'ensemble des secteurs jouent pour comprendre les trajectoires des salariés agricoles : le niveau de qualification supérieur favorise la stabilité en emploi ; un niveau plus élevé de capital humain spécifique au secteur augmente l'ancrage dans celui-ci. De même, un niveau local de chômage plus élevé conduit à plus de précarité. Quelques mécanismes spécifiques au secteur sont aussi mis en lumière. Ainsi, le manque de qualification n'est pas toujours synonyme d'instabilité en agriculture. Les femmes ont une plus grande probabilité de suivre une trajectoire d'emplois précaires que leurs homologues masculins. Elles ont néanmoins autant de chances de se stabiliser en agriculture, lorsque l'on tient compte de l'expérience dans le secteur. Nous apportons aussi des éléments allant dans le sens d'une adaptation du secteur agricole à la situation locale : lorsque la concurrence avec l'industrie existe, les employeurs agricoles offriraient plus d'emplois stables.

Ces premiers résultats sont à mettre en perspective avec les mutations à venir du secteur. Si l'ouverture du secteur à des repreneurs non-héritiers se poursuit, si le rôle de l'agriculture poursuit sa diversification (tourisme, fourniture de biens publics...), si les pratiques plus respectueuses de l'environnement prennent plus de place... alors de profondes évolutions sont à prévoir en matière de main-d'œuvre salariée. Va-t-on aller vers une plus grande précarisation, ou bien les formes de groupements d'employeurs vont-elles se développer pour sécuriser les parcours ? Allonsnous vers des emplois plus qualifiés ? Autant de questions qu'il convient d'aborder si l'on veut comprendre les enjeux du secteur agricole dans la décennie qui vient.

Remerciements : Les auteurs remercient Virginie Piguet pour la mise à disposition de sa matrice du test de Small et Hsiao, Éric Cahuzac, Marc Parmentier ainsi que les deux relecteurs anonymes pour leurs commentaires avisés. 


\section{RÉFÉRENCES BIBLIOGRAPHIQUES}

Battu H., Zenou Y. (2010). Oppositional identities and employment for ethnic minorities: evidence for England. The Economic Journal, ${ }^{\circ} 120$, p. 52-71.

Blanc M., Cahuzac E., Elyakime B (2008). Demand for on-farm permanent hired labour on family holdings. European Review of Agricultural Economics, vol. 35, $\mathrm{n}^{\circ} 4$, p. 493-518.

Bojnec S., Dries L. (2005). Causes of changes in agricultural employment in Slovenia: Evidence from micro-data. Journal of Agricultural Economics, vol. 56, $\mathrm{n}^{\circ} 3$, p. 399-416.

Boockmann B., Hagen T. (2008). Fixed-term contracts as sorting mechanisms: Evidence from job durations in West Germany. Labour Economics, vol. 15, p. 984-1005.

Burdett K. (1978). Employee Search and Quits. American Economic Review, n68, p. 212-220.

Cahuzac E., Détang-Dessendre C. (2011). Le salariat agricole : une part croissante dans l'emploi des exploitations, mais une précarité des statuts. Économie rurale, n ${ }^{\circ} 323$, p. 82-92.

Confais J., Nakache J.-P. (2000). Méthodes de classification. CISIA-CERESTA.

Davy F. (2012). Sécuriser les parcours professionnels par la création d'un compte social universel. Rapport pour le ministre du travail, de l'emploi et de la santé, avril 2012, Paris, La Documentation française, $40 \mathrm{p}$.

De Graaf-Zijl M., Van den Berg G. J., Heyma A. (2011). Stepping-stones for the unemployed: the effect of temporary jobs on the duration until (regular) work. Journal of Population Economics, vol. 24, $\mathrm{n}^{\circ} 1$, p. 107-139.

Errington A., Gasson R. (1994). Labour use in the farm family business. Sociologia ruralis, $\mathrm{n}^{\circ} 34$, p. 293-307.

Gabadinho A., Studer M., Müller N., Ritschard G. (2010). Mining sequence data in $R$ with the TraMineR package: A user's guide. Geneva, Department of Econometrics and Laboratory of Demography, University of Geneva, $100 \mathrm{p}$.

Gagliarducci S. (2005). The dynamics of repeated temporary jobs. Labour Economics, $\mathrm{n}^{\circ} 12$, p. 429-448.

Gaigné C. (2000). Appariement et stabilité de la relation d'emploi dans les espaces ruraux. Revue d'Économie régionale et urbaine, $\mathrm{n}^{\circ} 5$, p. 821-840.

Gullstrand J., Tezic K. (2008). Who leaves after entering the primary sector? Evidence from Swedish micro-level data. European Review of Agricultural Economics, vol. 35, $\mathrm{n}^{\circ} 1$, p. 1-28.

Harris J. R., Todaro M. (1970). Migration, unemployment and development: A twosector analysis. American Economic Review, $\mathrm{n}^{\circ} 60$, p. 126-142.

Hausman J., McFadden D. (1984). Specification Tests for the Multinomial Logit Model. Econometrica, vol. 52, n 5, p. 1219-1240.

Hervieu B., Purseigle F. (2013). Sociologie des mondes agricoles, Paris, Armand Colin, coll. «U Sociologie », 318 p.

Jovanovic B. (1979). Job matching and theory of turnover. Journal of political Economy, $\mathrm{n}^{\circ} 87$, p. 972-990.

Jovanovic B. (1984). Matching, turnover and unemployment. Journal of political Economy, n 92, p. 108-122.

Julien P., Pougnard J. (2004). Les bassins de vie, au cour de la vie des bourgs et des petites villes. Insee Première, p. 953.

Lippman S. A, McCall J. (1976). The economics of job search: a survey. Economic Inquiry, vol. $14, \mathrm{n}^{\circ} 2$, p. $155-189$ et p. 347-368

Mincer J., Ofek H. (1982). Interrupted Work Careers: Depreciation and Restoration of Human Capital. Journal of Human Resources, vol. 17, $\mathrm{n}^{\circ}$ 1, p. 1-24.

Narendranathan W., Elias P. (1993). Influences of Past History on the Incidence of Youth Unemployment: Empirical Findings for the UK. Oxford Bulletin of Economics and Statistics, Department of Economics, University of Oxford, vol. 55, $\mathrm{n}^{\circ} 2$, p. 161-85. 
RECHERCHES

Sonia BELLIT, Cécile DÉTANG-DESSENDRE

Palheta U., Gehin J.-P. (2012). Les devenirs socioprofessionnels des sortants sans diplômes. Un état des lieux dix ans après la sortie du système éducatif (1998-2008). Formation Emploi, $\mathrm{n}^{\circ} 118$, p. 15-35.

Peltier C., Marguet J., Privat C., Coulombel A. (2009). La place du travail salarié dans la gestion des exploitations agricoles. NESE, $n^{\circ} 32$, p. 41-59.

Pharo P., Schaff J.-P., Simula P. (1981). Les emplois de salariés agricoles. Paris, CEREQ, Ministère Éducation nationale.

Robette N. (2011). Explorer et décrire les parcours de vie : les typologies de trajectoires. CEPED (les Clefs pour...), $86 \mathrm{p}$.

Rogerson R., Shimer R., Wright R. (2005). Search-theoretic models of the labor market: a survey. Journal of Economic Literature 43 (4), 959-988.

Rohwer G., Pötter U. (2005). TDA's user manual. En ligne : http://www.stat.ruhr-unibochum.de/tman.html.

Small K. A., Hsiao C. (1985). Multinomial logit specification tests. International Review, ${ }^{\circ} 26$, p. 619-627.
Thisse J., Zenou Y. (1995). Appariement et concurrence spatiale sur le marché du travail. Revue économique, $\mathrm{n}^{\circ}$ 43, p. 615-624.

Thomas A. (2000). Économétrie des variables qualitatives. Paris, Dunod, $179 \mathrm{p}$.

Van den Berg G. (1992). A Structural dynamic analysis of Job Turnover and the Costs Associated with Moving to Another Job. The Economic Journal, $\mathrm{n}^{\circ} 102$, p. 1116-1133.

Van den Berg G., Holm A., van Ours J. (2002). Do stepping-stone jobs exist? Early career paths in the medical profession. Journal of Population Economics, ${ }^{\circ}$ 15, p. 647-665.

Van Ours J. C. (2004). The locking-in effect of subsidized jobs. Journal of Comparative Economics, $\mathrm{n}^{\circ} 32$, p. 37-55.

Wallace C., Dunderley D., Cheal B., Warren M. (1994). Young people and the division of labour in farming families. The Sociological Review, ${ }^{\circ} 42$, p. 501-530.

Zenou Yves (2009). Urban Labor Economics. Cambridge University Press, 509 p. 


\section{ANNEXE}

Tableau A.1. Description de l'échantillon et des trajectoires-types

\begin{tabular}{|c|c|c|c|c|c|c|c|c|}
\hline & $\begin{array}{l}\text { Stable } \\
\text { en agri. }\end{array}$ & $\begin{array}{l}\text { Stable } \\
\text { hors } \\
\text { agri. }\end{array}$ & $\begin{array}{l}\text { Stable } \\
\text { com- } \\
\text { biné }\end{array}$ & $\begin{array}{c}\text { Saison- } \\
\text { nier en } \\
\text { agri. }\end{array}$ & $\begin{array}{l}\text { Instable } \\
\text { hors } \\
\text { agricul- } \\
\text { ture }\end{array}$ & $\begin{array}{l}\text { Instable } \\
\text { en agri. }\end{array}$ & $\begin{array}{l}\text { Chô- } \\
\text { meurs } \\
\text { récur- } \\
\text { rents }\end{array}$ & Total \\
\hline \multicolumn{9}{|l|}{ Sexe } \\
\hline Homme & $69,33 \%$ & $62,34 \%$ & $63,91 \%$ & $49,36 \%$ & $54,65 \%$ & $58,76 \%$ & $53,49 \%$ & $59,86 \%$ \\
\hline Femme & $30,67 \%$ & $37,66 \%$ & $36,09 \%$ & $50,64 \%$ & $45,35 \%$ & $41,24 \%$ & $46,51 \%$ & $40,14 \%$ \\
\hline \multicolumn{9}{|l|}{ Tranche d'âge } \\
\hline $28-39$ ans & $47,38 \%$ & $54,55 \%$ & $51,13 \%$ & $44,23 \%$ & $65,12 \%$ & $52,58 \%$ & $49,61 \%$ & $49,62 \%$ \\
\hline 40-49 ans & $38,90 \%$ & $31,17 \%$ & $35,34 \%$ & $40,38 \%$ & $25,58 \%$ & $29,90 \%$ & $31,78 \%$ & $35,75 \%$ \\
\hline $50-59$ ans & $12,97 \%$ & $12,99 \%$ & $11,28 \%$ & $11,22 \%$ & $8,14 \%$ & $14,43 \%$ & $14,73 \%$ & $12,35 \%$ \\
\hline 60 ans et plus & $0,75 \%$ & $1,30 \%$ & $2,28 \%$ & $4,17 \%$ & $1,16 \%$ & $3,09 \%$ & $3,88 \%$ & $2,29 \%$ \\
\hline \multicolumn{9}{|l|}{ Nationalité } \\
\hline Français & $84,04 \%$ & $83,77 \%$ & $86,47 \%$ & $75,64 \%$ & $86,05 \%$ & $71,13 \%$ & $81,40 \%$ & $81,17 \%$ \\
\hline Étranger & $4,49 \%$ & $12,99 \%$ & $3,76 \%$ & $17,63 \%$ & $10,47 \%$ & $12,37 \%$ & $13,18 \%$ & $10,37 \%$ \\
\hline Inconnu & $11,47 \%$ & $3,25 \%$ & $9,77 \%$ & $6,73 \%$ & $3,49 \%$ & $16,49 \%$ & $5,43 \%$ & $8,46 \%$ \\
\hline \multicolumn{9}{|l|}{ PCS } \\
\hline Ouvriers non qualifiés & $46,64 \%$ & $19,48 \%$ & $33,08 \%$ & $52,88 \%$ & $12,79 \%$ & $49,48 \%$ & $54,94 \%$ & $42,07 \%$ \\
\hline $\begin{array}{l}\text { Ouvriers qualifiés - } \\
\text { employés }\end{array}$ & $34,91 \%$ & $59,09 \%$ & $48,87 \%$ & $38,79 \%$ & $74,42 \%$ & $37,11 \%$ & $40,31 \%$ & $43,37 \%$ \\
\hline $\begin{array}{l}\text { Cadres et professions } \\
\text { intermédiaires }\end{array}$ & $18,45 \%$ & $21,43 \%$ & $18,05 \%$ & $8,33 \%$ & $12,79 \%$ & $13,40 \%$ & $7,75 \%$ & $14,56 \%$ \\
\hline $\begin{array}{l}\text { Expérience moy. en agri. } \\
\text { Jan } 2002 \text { (mois) : }\end{array}$ & 49 & 14 & 28 & 15 & 5 & 26 & 16 & 27 \\
\hline \multicolumn{9}{|c|}{ Taux d'emploi par secteur du BV (1) } \\
\hline Agricole & $11,69 \%$ & $11,28 \%$ & $11,71 \%$ & $12,21 \%$ & $10,57 \%$ & $11,12 \%$ & $11,70 \%$ & $11,66 \%$ \\
\hline Industriel & $20,56 \%$ & $18,22 \%$ & $19,75 \%$ & $17,54 \%$ & $19,12 \%$ & $18,65 \%$ & $19,25 \%$ & $19,12 \%$ \\
\hline Services & $57,60 \%$ & $60,27 \%$ & $58,11 \%$ & $59,64 \%$ & $59,76 \%$ & $59,87 \%$ & $58,88 \%$ & $58,89 \%$ \\
\hline \multicolumn{9}{|c|}{ Orientation agricole du BV (1) } \\
\hline Cultures permanentes & $12 \%$ & $12,34 \%$ & $17,29 \%$ & $26,60 \%$ & $7 \%$ & $13,40 \%$ & $17,83 \%$ & $16,39 \%$ \\
\hline Grandes cultures & $16,21 \%$ & $17,53 \%$ & $15,79 \%$ & $12,82 \%$ & $12,79 \%$ & $17,53 \%$ & $13,18 \%$ & $15,09 \%$ \\
\hline $\begin{array}{l}\text { Herbivores (bovins, } \\
\text { ovins, caprins, équidés) }\end{array}$ & $15,96 \%$ & $12,34 \%$ & $19,55 \%$ & $6,09 \%$ & $16,28 \%$ & $6,19 \%$ & $10,85 \%$ & $12,35 \%$ \\
\hline $\begin{array}{l}\text { Horticultures, } \\
\text { maraîchage }\end{array}$ & $5,49 \%$ & $1,95 \%$ & $0,75 \%$ & $1,92 \%$ & $2,33 \%$ & $7,22 \%$ & $2,33 \%$ & $3,35 \%$ \\
\hline Granivores & $4,74 \%$ & $3,25 \%$ & $3,76 \%$ & $0,32 \%$ & $3,49 \%$ & $2,06 \%$ & $3,88 \%$ & $3,05 \%$ \\
\hline $\begin{array}{l}\text { Taux de chômage } \\
\text { moyen du BV en } 99\end{array}$ & $11,77 \%$ & $12,73 \%$ & $11,68 \%$ & $13,42 \%$ & $12,43 \%$ & $12,98 \%$ & $12,97 \%$ & $12,52 \%$ \\
\hline $\begin{array}{l}\text { Dist. au pôle urbain } \\
\text { le plus proche (mn) }\end{array}$ & 23 & 23 & 24 & 23 & 24 & 22 & 23 & 23 \\
\hline $\begin{array}{l}\text { Dist. à l'UU de } 50000 \text { hab. } \\
\text { la plus proche }(\mathrm{mn}) \text { : }\end{array}$ & 36 & 34 & 35 & 35 & 34 & 33 & 36 & 35 \\
\hline Effectifs & 401 & 154 & 133 & 312 & 86 & 97 & 129 & 1312 \\
\hline
\end{tabular}

Note : (1) BV : Bassin de vie. 